\title{
GENERALIZATION OF SCARPIS'S THEOREM ON HADAMARD MATRICES
}

\author{
DRAGOMIR Ž. ĐOKOVIĆ
}

\begin{abstract}
A $\{1,-1\}$-matrix $H$ of order $m$ is a Hadamard matrix if $H H^{T}=$ $m I_{m}$, where $T$ is the transposition operator and $I_{m}$ the identity matrix of order $m$. J. Hadamard published his paper [1] on Hadamard matrices in 1893. Five years later, Scarpis 4 showed how one can use a Hadamard matrix of order $n=1+p, p \equiv 3(\bmod 4)$ a prime, to construct a bigger Hadamard matrix of order $p n$. In this note we show that Scarpis's construction can be extended to the more general case where $p$ is replaced by a prime power $q$.
\end{abstract}

\section{INTRODUCTION}

We fix some notation which will be used throughout this note. By $\mathcal{H}_{m}$ we denote the set of Hadamard matrices of order $m$. Let $q \equiv 3(\bmod 4)$ be a prime power and set $n=1+q$. Let $F_{q}$ be a finite field of order $q$.

Given a bijection $\alpha:\{1,2, \ldots, q\} \rightarrow F_{q}$, we shall construct a map

$$
\varphi_{q, \alpha}: \mathcal{H}_{n} \rightarrow \mathcal{H}_{q n}
$$

Consequently, the following theorem holds.

Theorem 1. Let $q \equiv 3(\bmod 4)$ be a prime power. If there exists a Hadamard matrix of order $n=1+q$ then there exists also a Hadamard matrix of order qn.

In the special case, where $q$ is a prime, this theorem was proved by Scarpis 4 . For a nice and short description of the original Scarpis's construction see [2].

By the well known theorem of Paley [3], the hypothesis of the above theorem is always satisfied. Thus we have

Corollary 1. If $q \equiv 3(\bmod 4)$ is a prime power, then there exists a Hadamard matrix of order $q(1+q)$.

We shall describe a procedure whose input is a Hadamard matrix $A=\left[a_{i, j}\right]$ of order $n=1+q$ and output a Hadamard matrix $B=\varphi_{q, \alpha}(A)$ of order $q n$. For convenience, we set $\alpha_{i}=\alpha(i)$.

\section{Construction of $B$}

Step 1: If $a_{1,1}=-1$ then replace $A$ by $-A$. From now on $a_{1,1}=1$.

Step 2: For each $i \in\{2,3, \ldots, n\}$ do the following: if $a_{i, 1}=-1$ then multiply the row $i$ of $A$ by -1 , and if $a_{1, i}=-1$ then multiply the column $i$ of $A$ by -1 . The resulting matrix $A$ is independent of the order in which these operations are performed.

Note that $A$ is now normalized, i.e., $a_{i, 1}=a_{1, i}=1$ for each $i$. Denote by $C$ its core, i.e., the submatrix of $A$ obtained by deleting the first row and the first column 
of $A$. For $i \in\{1,2, \ldots, q\}$, we denote by $c_{i}$ the row $i$ of $C$. For convenience, we also set $c\left(\alpha_{i}\right)=c_{i}$.

The tensor product $X \otimes Y$ of two matrices $X=\left(x_{i, j}\right)$ and $Y$ is the block matrix $\left[x_{i, j} Y\right]$.

Let $\mathbf{j}$ be the row vector of length $q$ all of whose entries are 1 . We view $\mathbf{j}$ also as a $1 \times q$ matrix.

Step 3: We partition $B$ into $n$ blocks of size $q \times q n$ :

$$
B=\left[\begin{array}{c}
B_{0} \\
B_{1} \\
\vdots \\
B_{q}
\end{array}\right]
$$

We set $B_{0}=A^{\prime} \otimes \mathbf{j}$ where $A^{\prime}$ is the submatrix of $A$ obtained by deleting the first row of $A$.

Step 4: For $r \in\{1,2, \ldots, q\}$, we partition $B_{r}$ into $n$ blocks of size $q \times q$ :

$$
B_{r}=\left[\begin{array}{llll}
B_{r, 0} & B_{r, 1} & \cdots & B_{r, q}
\end{array}\right] .
$$

We set $B_{r, 0}=\mathbf{j}^{T} \otimes c_{r}$.

It remains to define the blocks $B_{r, i}$ for $\{r, i\} \subseteq\{1,2, \ldots, q\}$.

Step 5: For $\{r, i\} \subseteq\{1,2, \ldots, q\}$, we define $B_{r, i}$ by specifying that its row $k$ is $c\left(\alpha_{i} \alpha_{r}+\alpha_{k}\right)$. Thus $B_{r, i}=P_{r, i} C$ where $P_{r, i}$ is a permutation matrix.

This completes the definition of $B$.

It remains to prove that $B$ is a Hadamard matrix.

\section{Proof that $B$ is a Hadamard matrix}

As $B$ is a square $\{1,-1\}$-matrix of order $q n$, it suffices to prove that the dot product of any pair of rows of $B$ is 0 . There are four cases to consider.

(i) Two distinct rows of $B_{0}$. They are orthogonal because two distinct rows of $A^{\prime}$ are orthogonal.

(ii) Two distinct rows of $B_{r}, r \neq 0$. Since $A$ is normalized, the dot product $c_{r} \cdot c_{s}$ is $q$ when $r=s$, and -1 otherwise. Hence, the same is true for each of the blocks $B_{r, i}$ for $i \neq 0$. On the other hand, the dot product of any two rows of $B_{r, 0}$ is $q$. It follows that the dot product of any pair of rows of $B_{r}$ is 0 .

(iii) A row of $B_{0}$ and a row of $B_{s}, s \neq 0$.

The row $k$ of $B_{0}$ is $\left[\mathbf{j} \quad c_{k} \otimes \mathbf{j}\right]$ and the row $l$ of $B_{s}$ is

$$
\left[c\left(\alpha_{s}\right) c\left(\alpha_{s} \alpha_{l}+\alpha_{1}\right) c\left(\alpha_{s} \alpha_{l}+\alpha_{2}\right) \cdots c\left(\alpha_{s} \alpha_{l}+\alpha_{q}\right)\right] .
$$

Since all row sums of $C$ are -1 , it follows that the dot product of the two rows above is 0 .

(iv) A row of $B_{r}$ and a row of $B_{s}, 0<r<s$.

The dot product of the row $k$ of $B_{r}$ and the row $l$ of $B_{s}$ is

$$
c\left(\alpha_{r}\right) \cdot c\left(\alpha_{s}\right)+\sum_{i=1}^{q} c\left(\alpha_{i} \alpha_{r}+\alpha_{k}\right) \cdot c\left(\alpha_{i} \alpha_{s}+\alpha_{l}\right) .
$$

Note that $c\left(\alpha_{i} \alpha_{r}+\alpha_{k}\right) \cdot c\left(\alpha_{i} \alpha_{s}+\alpha_{l}\right)$ is equal to -1 for all $i$ except that it is equal to $q$ for the unique $i \in\{1,2, \ldots, q\}$ for which $\alpha_{i} \alpha_{r}+\alpha_{k}=\alpha_{i} \alpha_{s}+\alpha_{l}$. Since also $c\left(\alpha_{r}\right) \cdot c\left(\alpha_{s}\right)=-1$, it follows that the rows of $B_{r}$ are orthogonal to the rows of $B_{s}$. 
We have shown that $B \in \mathcal{H}_{q n}$. This completes our construction of $\varphi_{q, \alpha}$.

The smallest $q$ which satisfies the condition of Theorem 1 but is not a prime (so Scarpis's theorem does not apply) is $q=27$. It gives a Hadamard matrix of order $4 \cdot 189=756$.

We conclude with an open problem: Find an analog of our procedure which uses prime powers $q \equiv 1(\bmod 4)$.

The author acknowledges generous support by NSERC.

\section{REFERENCES}

[1] J. Hadamard, Résolution d'une question relative aux déterminants, Bull. des Sciences Mathématiques 17 (1893) 240-246.

[2] W. Orrick, Maximal determinant blog, Hadamard matrices: the construction of Scarpis. //willorrick.wordpress.com/2012/11/17/ November 17, 2012

[3] R. E. A. C. Paley, On orthogonal matrices, Journal of Mathematics and Physics 12 (1933) 311-320.

[4] U. Scarpis, Sui determinanti di valore massimo, Rendiconti della R. Istituto Lombardo di Scienze e Lettere (2), 31 (1898) 1441-1446.

University of Waterloo, Department of Pure Mathematics and Institute for Quantum Computing, Waterloo, Ontario, N2L 3G1, Canada

E-mail address: djokovic@uwaterloo.ca 